\title{
Numerical Calculation and Analysis of Magnetic Source Transient Electromagnetic Field in Stratum Medium
}

\author{
Qingxin MENG \\ College of exploration and Engineering \\ Shijiazhuang University of Economics \\ Shijiazhuang, China \\ e-mail: moskit850@aliyun.com
}

\author{
Zujian LIU \\ College of exploration and Engineering \\ Shijiazhuang University of Economics \\ Shijiazhuang, China \\ e-mail: dasliuzj@gmail.com
}

\begin{abstract}
The electromagnetic component expressions of transient electromagnetic field in formation media for ground magnetic dipole are derived based on electromagnetic field theory. Fast Hankel transform and the Gaver-Stehfest inverse Laplace transform method is used for forward numerical calculation of transient electromagnetic field in a uniform earth and layered medium. The regularity and characteristics of surface-hole transient electromagnetic method response was analyzed base on forward simulations in different conditions. The result shows that, the surfacehole TEM magnetic induction response sign change occur if borehole located farther offset distance, the status of signchange would alter in different time channel and different offset distance conditions, the induced electromotive force response can directly reflect the changes of induced eddy current field in formation medium, there is obvious difference in the electromagnetic response strength and attenuation characteristics for different electrical formation media, the difference can be used as the basis of inference layered ground dielectric structure.
\end{abstract}

Keywords-surface-hole TEM; magnetic dipole source; layered medium; half-space of homogeneous

\section{INTRODUCTION}

Surface-borehole TEM (Surface-borehole transient electromagnetic method) usually adopts the loop source laid on the ground for pulse current to generate the pulse current. After the field source is cut off, the probe in the borehole receives the secondary field generated by formation and target induction. Through research and analysis of features of the secondary field, the information about the target beside the borehole (at the bottom of the borehole) is gained. Since surface-borehole TEM mainly works through analyzing the rule of response characteristics, forward modeling becomes a significant issue. Literature [1] applies circulation method to calculate surface-borehole TEM response and summarizes the interpretation means. Literatures [2]-[4] discuss response sign changes of surface-borehole TEM in conductive surrounding rock, effects on observed results and observed result signal reception. Literature [5] completes physical experiment of surface-borehole TEM under typical conducting medium conditions. Literature [6] presents combined application of TEM on the surface and in the borehole. Literatures [7]-[8] study combined retrieval method for TEM data on the surface and in the borehole. Chinese researchers discuss interpretation principle of surface-borehole TEM based on equivalent eddy [9]-[10]. Literatures [11]-[12] carry out researches in terms of numerical simulation and practical application and gain certain achievements in mine TEM [13]-[14].

In practical work, formation media (including homogeneous half-space medium, low-resistance covering layer and multi-layered medium) will influence observed results of surface-borehole TEM. The signals received are restrained by multiple factors, and the rule of response characteristics is relatively complex. On the other hand, transient electromagnetic field generated by magnetic dipole source in certain space and late time channel is homogeneous and stable. Characteristic rule of the electromagnetic field is somewhat similar to the loop source. Besides, it is simpler and more vivid in the aspect of discussing TEM forward modeling with non-zero offset distance. This paper applies magnetic dipole source TEM numerical calculation method to carry out 3D forward modeling for the background field (transient field generated by excitation source of ground magnetic dipole in formation medium) and analyzes the rule of response characteristics so as to provide reference for relevant studies.

\section{TRANSIENT ELECTROMAGNETIC FIELD GENERATED UNDER THE GROUND BY GROUND MAGNETIC DIPOLE SOURCE}

\section{A. harmonic fields formed under the ground by ground vertical magnetic dipole source}

Assuming horizontally layered and isotropous linear medium, cylindrical coordinate system is set up; displacement current is neglected; vertical magnetic dipole source is located on the ground $\left(z=h_{0}\right)$; the particular solution of vertical vector component $A_{z}$ meets basic differential equation:

$$
\nabla^{2} A_{z_{j}}-k_{j}^{2} A_{z_{j}}=0
$$

In allusion to different layers, the solution is as follows: 


$$
\left\{\begin{array}{c}
A_{z_{j}}=\frac{i \omega \mu_{0} M}{4 \pi} \int_{0}^{\infty}\left(D_{j} e^{\lambda_{j} z}+C_{j} e^{-\lambda_{j} z}\right) J_{0}(\lambda r) d \lambda \\
A_{z_{j+1}}=\frac{i \omega \mu_{0} M}{4 \pi} \int_{0}^{\infty} C_{j+1} e^{-\lambda_{j+1} z} J_{0}(\lambda r) d \lambda
\end{array}\right.
$$

According to the continuity of electromagnetic field on each interface, the interface at each layer meets boundary conditions:

$$
\left\{\begin{array}{c}
A_{z_{j}}=A_{z_{j+1}} \\
\partial A_{z_{j}} / \partial z=\partial A_{z_{j+1}} / \partial z
\end{array}\right.
$$

The coefficients [10] $C_{n} 、 D_{n}(n=0,1, \cdots, j+1)$ can be solved in combination of Formula (2). Electric component (tangential direction) and magnetic component (radial direction/vertical direction) of layered formation can be solved according to Formula (4). After simplification, the solutions of each field quantity in homogeneous half-space can be gained.

$$
\left\{\begin{array}{c}
E_{\varphi}=-\frac{\partial A_{z}}{\partial r} \\
i \omega \mu_{0} H_{z}=-\frac{1}{r} \frac{\partial}{\partial r}\left(r \frac{\partial A_{z}}{\partial r}\right)
\end{array}\right.
$$

In accordance with the result of Formula (4), the solutions of each field component can be further obtained. In Formula (1) - (4), magnetic moment $M=S_{T_{X}} n_{T_{x}} I_{T x}$, where $r$ is offset distance; $\mu_{0}$ is magnetic conductivity; $\sigma_{j}$ is the conductivity of the $j^{\text {th }}$ layer; $k_{j}^{2}=-i \omega \mu_{0} \sigma_{j}$ and $\lambda_{j}^{2}=\lambda^{2}-k_{j}^{2}$.

\section{B. Transient response numerical calculation based on \\ Fast Hankel Transform and G-S Inverse Laplace \\ Transform}

Formula (2) is typical Hankel integral. Predecessors did a lot of work on numerical algorithm and calculation precision related to the problem. This paper applies the FHT method. Hankel integral form and disperse convolution form are:

$$
\begin{gathered}
F(r)=\int_{0}^{\infty} h(\lambda) J_{n}(\lambda r) d r \\
F(r)=\left\{\sum_{i=n_{1}}^{n_{2}} H_{n, i} \cdot h\left[\exp \left(A_{i}-x\right)\right]\right\} / r
\end{gathered}
$$

In the formula, $H_{n, j}$ is Hankel Transform filtering weight coefficient. The following can be gained through selecting proper input and output functions and Fourier forward and reverse transformation: $A_{i-x}$ is mobile weight horizontal coordinate. The result of alternating electromagnetic field can be solved according to Formula (6).

With regard to numerical calculation from harmonic fields to transient fields, in view of heavy calculation amount for the whole section, Gaver-Stehfes probability transform algorithm as inverse Laplace transform.. In combination of the above formulas, this paper lists inverse Laplace transform formula with the field source as step current, G-S discrete expression and solution expressions of relevant transformation coefficient are as below:

$$
\begin{gathered}
f(t)=\frac{1}{2 \pi i} \int_{0}^{\infty} \frac{F(s)}{s} \cdot e^{s t} d s \\
f(t)=\frac{\ln 2}{t} \sum_{m=1}^{M} G_{m}\left\{F\left(\frac{\ln 2}{t} \cdot m\right) /\left(\frac{\ln 2}{t} \cdot m\right)\right\} \\
G_{m}=(-1)^{m+\frac{M}{2}} \cdot \sum_{k=\ln [(m+1)) / 2]}^{\min \left(m, \frac{M}{2}\right)} \frac{k^{M}(2 k) !}{(M-k) ! k !(k-1) !(j-k) !(2 k-j) !}
\end{gathered}
$$

The above algorithms can be used for 3D forward numerical calculation of magnetic dipole source transient electromagnetic field with layered medium and can also be used for initial numerical simulation calculation of 3D numerical simulation with FDTD method.

\section{FORWARD NUMERICAL CALCULATION OF TEM WITH MAGNETIC DIPOLE SOURCE}
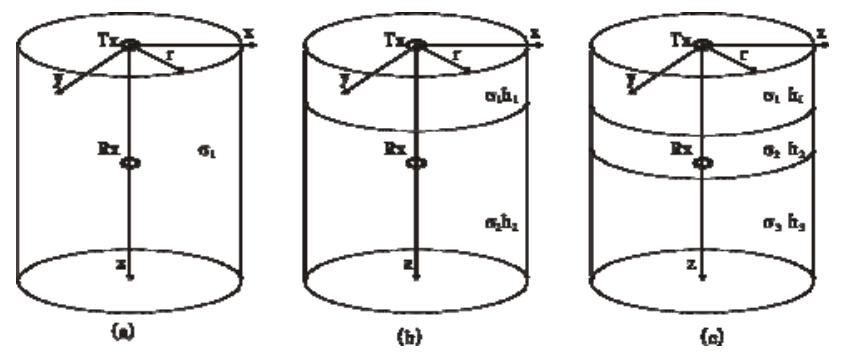

(c)

Figure 1. half-space 3-D model

Based on the previous calculation method, $\mathrm{C}$ programming is adopted for forward numerical calculation for transient magnetic field generated by ground magnetic dipole source under ground. This paper sets three types of basic geoelectric models: Fig.1-a: homogeneous earth conductivity $0.01 \mathrm{~S} / \mathrm{m}$; Fig.1-b: two-layer medium; the first layer is $100 \mathrm{~m}$ thick, with the conductivity of $1 \mathrm{~S} / \mathrm{m}$; the conductivity of the second layer is $0.01 \mathrm{~S} / \mathrm{m}$; Fig.1-c: threelayer earth medium; the first layer is $170 \mathrm{~m}$ thick, with conductivity of $0.01 \mathrm{~S} / \mathrm{m}$; the second layer is $80 \mathrm{~m}$ thick, with conductivity of $1 \mathrm{~S} / \mathrm{m}$; the conductivity of third layer is $0.01 \mathrm{~S} / \mathrm{m}$. In the figures, the medium is the rectangular coordinate system with three axial directions: $x$ (horizontal crosswise), $y$ (horizontal lengthways) and $z$ (vertical depth), where $r$ is the offset distance. Parameters of surfaceborehole TEM transmitter-receiver set: field source $T x$ is located in the origin $(x=0, y=0, z=0)$ with the area of $1 \mathrm{~m}^{2}$, 50 turns and current intensity of 50A; the receiving coil Rx is placed in vertical boreholes with different offset distance to receive axial component of the borehole responded by TEM. The observing depth is $20-400 \mathrm{~m}$ and measurement distance is $4 \mathrm{~m}$.

\section{A. TEM in homogeneous earth and surface-borehole response characteristics}

Fig.2 shows the contour of electric field component $\mathrm{E}$ of homogeneous earth $(0.01 \mathrm{~S} / \mathrm{m})$ TEM (Fig.2-a and b present vertical section contour with "horizontal $x$-depth $z$ "; Fig.2-c and d show the contour of horizontal section at the depth). From the figures, diffusion situation of magnetic dipole source TEM in the geoelectric model can be known: TEM generated by inductive eddy of homogeneous earth diffuses downward and outward as time delays; field intensity attenuates over time; the transient field is an eddy field including a loop-shaped maximum value ("smoke 
ring" shape) in 3D homogeneous half-space distribution [8].

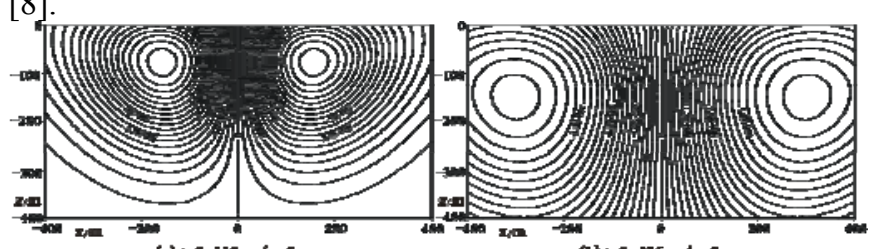

(Q) $=0.11 \operatorname{mon}_{3}=0$ an

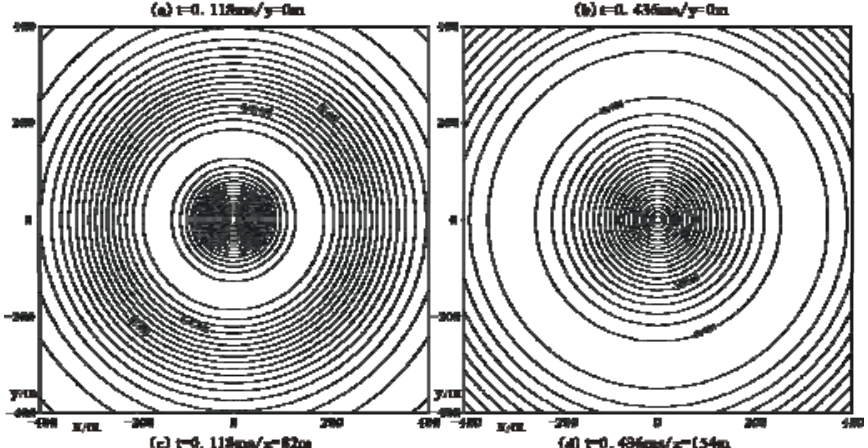

Figure 2. Electric field contour in half-space of homogeneous medium

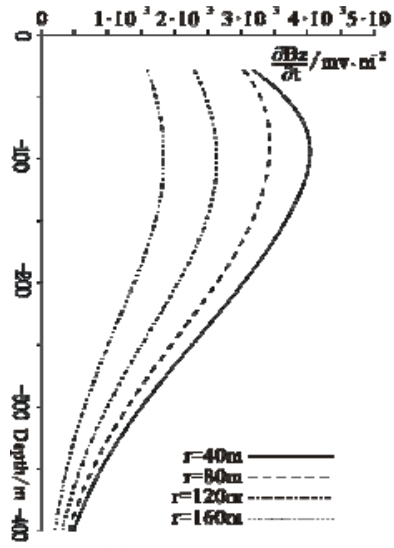

(a) $t=0.182 \mathrm{mos}$

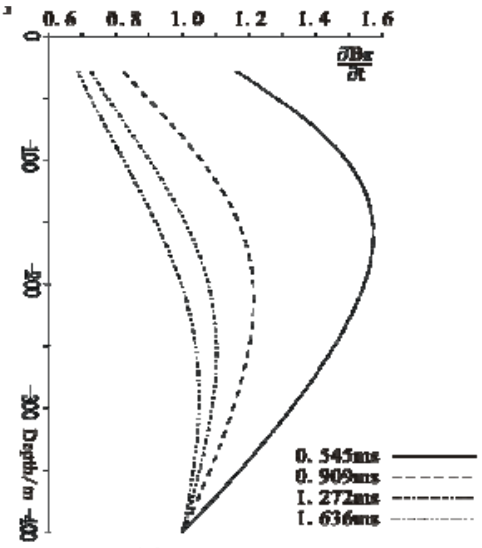

(b) $r=\operatorname{man}$
Figure 3. TEM responses for half-space of uniform medium $(\partial \mathrm{Bz} / \partial \mathrm{t})$

Fig.2-a and Fig.2-b present the contour of section electric field quantity at different time with $y=0$. On the section, $E x=0$ and visible electric field quantity is $E y$ component. Fig.2-c and Fig.2-d show the contours of section electric field intensity at different time under $z=82 \mathrm{~m}$ and $z=154 \mathrm{~m}$, respectively. The depth segment is roughly where the maximum value of electric field quantity of different depth sections in $3 \mathrm{D}$ space under the parameters of the model is located (field intensity in the figure is positive).

Fig.3-a shows the response curve of induced electromotive force $(\partial \mathrm{Bz} / \partial \mathrm{t})$ under homogenous half-space medium at $0.182 \mathrm{~ms}$. Referring to inductive eddy electric field distribution in Fig.2, the rule of response curve characteristics is roughly as follows: the intensity at superficial-layer measuring point is large; the intensity at deep-layer measuring point is small; the maximum response value received is distributed near the depth where the maximum value of eddy electric field is located. Fig.3b shows the curve after normalization of the response received at the deepest measuring point at different time under homogeneous half-space: the response range gradually reduces with time delay, which means the overall distribution of the induction field tends to be homogenous with time delay; the position of the maximum response value gradually deepens, which indicates that the maximum value of the induction field gradually moves down over time [12].

B. Response characteristics of TEM in layered earth
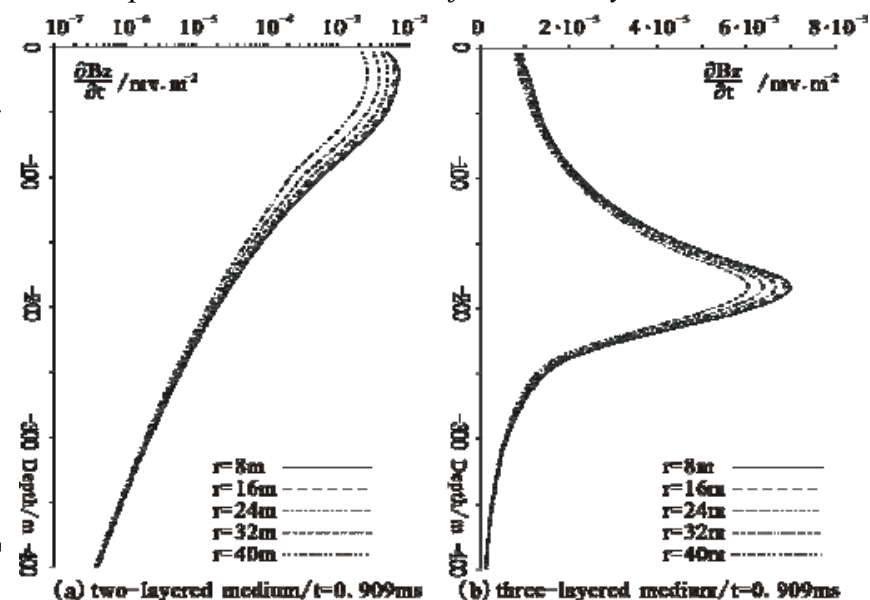

Figure 4. TEM responses for layered medium $(\partial \mathrm{Bz} / \partial \mathrm{t})$

Fig.4 shows the response curve of induced electromotive force $(\partial \mathrm{Bz} / \partial \mathrm{t})$ measured by surface-borehole TEM in layered medium. The response curve in the figure reflects layered structure feature of the geoelectric model: in Fig.4-a, the amplitude at superficial layer of the response curve is large, which shows good electrical conductivity of low-resistance covering layer. On the other hand, since electromagnetic wave propagation speed is influenced, the maximum value of inductive eddy field at this moment is in low-resistance layer (comparing Fig.3). In Fig.4-b, the response amplitude is high, because electrical conductivity of the second segment of formation is good and the maximum value of inductive eddy field at this moment is at the depth. On the curve, it can be obviously recognized that electrical conductivity of the middle layer is obviously higher than the upper and lower layers. It is necessary to explain that response curve feature shown in Fig.4 is the concurrent result of eddy field propagation change distribution in layered medium over time and the formation with good electrical conductivity. In practice, with different time delay, the curve forms are also different.

Fig.5 presents response changes with time at different depth in the three types of geoelectric models. The depth points selected can basically reflect the position of each layer of the three geoelectric models. The vertical coordinate of the curve is the response amplitude. The response experiences normalization treatment of the response intensity at the last moment. The horizontal coordinate is time (about $0.364 \mathrm{~ms} \sim 3.64 \mathrm{~ms}$ ). It can be seen from the figure that response attenuation of different geoelectric structures has significant differences. Overall attenuation amplitude of the response measured in layered medium is small (Fig.5-d and c). The attenuation speed is slower than that in homogenous earth. The differences in propagation velocity and attenuation of electromagnetic wave in the formation with good electrical conductivity and in the formation with high resistance influence response changes and attenuation features of all measuring points on the whole borehole segment (see the response curve at different layers in Fig. 5-b and c). 


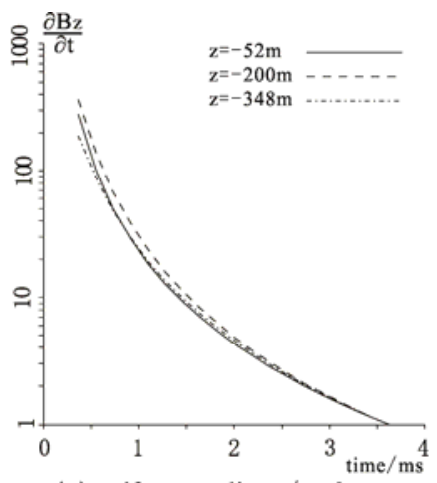

(a) uniform medium $/ \mathrm{r}=8 \mathrm{~m}$

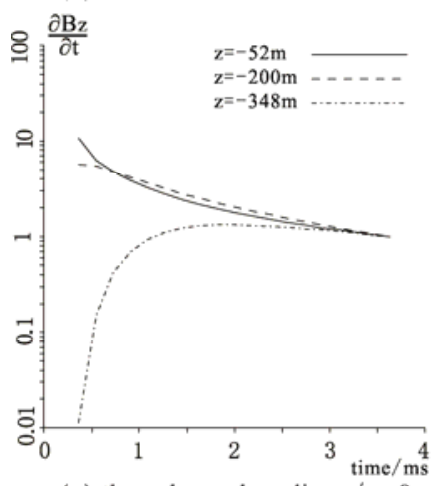

(c) three-layered medium $/ \mathrm{r}=8 \mathrm{~m}$

Figure 5. Attenuation of TEM responses for different layered medium at different depth

\section{CONCLUSIONS}

The electromagnetic component expressions of magnetic dipole surface-borehole TEM are derived based on electromagnetic field theory. Fast Hankel Transform and the Gaver-Stehfest Inverse Laplace Transform method are used for forward numerical calculation of transient electromagnetic field in the layered medium and homogeneous medium. Distribution change of underground transient field and measurement response of surface-borehole TEM were simulated. Besides, the rule of response characteristics was simply analyzed. The following results and conclusions are gained:

1) The response curve of induced electromotive force $(\partial \mathrm{Bz} / \partial \mathrm{t})$ measured by Surface-borehole TEM can visually reflect attenuation of underground inductive eddy field with time and variations of field intensity at different depth.

2) In the layered medium, electromagnetic response in the formation with different electrical property has differences in field intensity and attenuation features. In accordance with vertical section curve characteristics of surface-borehole TEM measurement response and attenuation situations with time delay, layered geoelectric medium structure can be inferred, and the layering position can be recognized under certain conditions.

3) Compared with uniform earth medium, differences exist between attenuation amplitude and attenuation speed of the response at each measuring point in the whole borehole segment in the layered medium, which can serve as the basis of inferring geoelectric structure.
Surface-borehole TEM response characteristics are complex and have many problems. The author only takes into account of fundamental characteristic rule of the response measured with magnetic source surface-borehole TEM. In practice work, large loop line is adopted as the excitation source. It is necessary to further study 3D forward modeling problem of loop source surface-borehole TEM measurement. Moreover, 3D forward numerical calculation amount is huge. The numerical calculation method in this paper needs to improve and boost the efficiency so as to adapt fast numerical simulation work.

\section{ACKNOWLEDGMENT}

Project supported by the Young Scientists Fund of the Natural Science Foundation of Hebei Province (Grant No. D2014403011) and the Young Scientists Fund of the National Natural Science Foundation of China (Grant No. 41304082).

\section{REFERENCES}

[1] A. V. Dyck and G. F. West, The role of simple computer models in interpretation of wide-band, drill-hole electromagnetic surveys in mineral exploration. Geophysics, 1984, Vol. 49, pp. 957 980.

[2] J. Macnae and G. Staltari, Classification of sign changes in Borehole TEM decays. Exploration Geophysics, 1987, Vol. 18, pp.331 339.

[3] A. P. Annan, The equivalent source method for electromagnetic scattering analysis and its geophysical application. Ph.D. thesis, Memorial University of Newfoundland, 1974, pp.242.

[4] P. R. Gallagher, S. H. Ward and G. W. Hohmann, A model study of a thin plate in free space for EM37 transient electromagnetic system. Geophysics, 1985, 50(6), pp.1002 1005.

[5] P. A. Eaton and G. W. Hohmann, The influence of a conductive host on two-dimensional borehole transient electromagnetic responses. Geophysics, 1984, Vol.49, pp. 861 869.

[6] R. C. West and S. H. Ward, The borehole transient EM of the three-dimensional fractured zone in a conductive half space. Geophysics, 1988, 53(11), pp.1469 1487.

[7] G. Buselli and S. K. Lee, Modelling of drill-hole TEM responses form multiple targets covered by a conductive overburden. Exploration Geophysics, 1996. Vol 27, pp.141 153.

[8] B. Y. Jiang, Applied Near Zone Magnetic Source Transient Electromagnetic Exploration. Beijing: Geological Publishing House, 1998.

[9] Z. L. Niu, The Theory of Time Domain Electromagnetic Methods. Changsha: Central South University of Technology Press, 2007.

[10] X. Li, The Theory and Application of Transient Electromagnetic Sounding . Xi'an: Shanxi Science Technology Press, 2002.

[11] J. D. Liu, Fang Wen-zao. The calculation of the TEM field underground generated by a large loop on the ground by means of the linear digital filter method. Computing techniques for geophysical and geochemical exploration, 1996, 18(3): pp.231-237.

[12] Meng Qing-xin, Pan He-ping. Numerical simulation analysis of surface-hole TEM responses [J]. Chinese J. Geophys. (in Chinese), 2012, 55(3): 1046-1053

[13] Ding Shi-rong, Fang Wen-zao. The transient electromagnetic response of a spherical geologic body excited by a step magnetic dipole [J]. Journal of Liao-ning Normal University (Natural Science), 1997, 20(3): 218-223.

[14] Chen Xi-jie, Ren Huai-zong. Response of a Buried Equiaxial Conductive Body in Drill Hole Transient Electromagnetic Measurements [J]. Geology and Prospecting, 1988, 24(11): 39-43.

[15] Zhang Sui-quan. An approximate formale for calculating EM transient reponse of a conducting sheet-like body [J]. Geology and Prospecting, 1990,26(9):35-40.

[16] Kaufman A A, Keller G V. Frequency and Transient Electromagnetic Soundings [M]. Beijing: Geological Publishing House, 1987. 